\title{
Association between a metabolic syndrome score and high sensitivity C-reactive protein in European children: the IDEFICS study
}

\author{
$\underline{\text { Esther M. Gonzalez-Gil }}^{1,2}$, Annunziata Nappo ${ }^{3}$, Javier Santabarbara ${ }^{4}$, Maike Wolters ${ }^{5}$, \\ Paola Russo $^{3}$, Stefaan De Henauw ${ }^{6}$, Toomas Veidebaum ${ }^{7}$, Denes Molnar ${ }^{8}$, Wolfgang Ahrens ${ }^{5}$ \\ and Luis Moreno ${ }^{1}$ \\ ${ }^{1}$ GENUD (Growth, Exercise, Nutrition and Development) Research Group, University of Zaragoza., Zaragoza, Spain, \\ ${ }^{2}$ Institute of Nutrition and Food Technology. Center of Biomedical Research. University of Granada, Granada, Spain, \\ ${ }^{3}$ Unit of Epidemiology and Population Genetics, Institute of Food Sciences, National Research Council, Avellino, Italy, \\ ${ }^{4}$ Department of Preventive Medicine and Public Health, University of Zaragoza, Zaragoza, Spain, \\ ${ }^{5}$ Leibniz Institute for Prevention Research and Epidemiology., Bremen, Germany, \\ ${ }^{6}$ Department of Public Health, Faculty of Medicine and Health Sciences, Ghent University, Ghent, Belgium, \\ ${ }^{7}$ National Institute for Health Development, Center of Health and Behavioral Science, Tallin, Estonia and \\ ${ }^{8}$ Department of Pediatrics, Medical School, University of Pécs, Pecs, Hungary
}

\begin{abstract}
Introduction: Metabolic syndrome (MetS) is a combination of risk factors that may be present already in childhood. MetS has been associated with inflammatory biomarkers such as high sensitivity C-reactive protein (hsCRP) in aduls. In 2014, Ahrens et al, published reference standards for a paediatric MetS score based on reference values from European children. The aim of this study is to assess longitudinally the relationship between a MetS score and hsCRP in a sample of European children.

Materials and Methods: Out of the baseline sample of the IDEFICS Study, 2913 children aged 2-9 years were included in this study. Inclusion criteria was having available data of waist circumference (WC), diastolic and systolic blood pressure (DBP, SBP), high density lipoprotein (HDL) cholesterol, triglycerides (TG), glucose and insulin, to calculate the homeostasis model assessment index (HOMA); and hs-CRP as a marker of inflammation, at baseline (T0) and two years later (T1). hs-CRP was categorized into two categories as some children had lower concentration than the detection limit of $0.02 \mathrm{mg} / \mathrm{dL}$. Student t-test and logistic regression were used to assess these associations. Logistic regression was adjusted by age, sex, body mass index (BMI), socioeconomic level and country.

Results: Differences of mean values of the components of the MetS and the two categories of hs-CRP were observed between both time points. Mean values of SBP, DBP, WC, TG and HOMA were significantly higher in children with a higher category of hsCRP $(p<0.005)$. In addition, MetS score was significantly higher in those with a higher category of hs-CRP $(p<0.001)$ at both measurement points, T0 and T1. Finally, logistic regression between components of MetS and categories of hs-CRP, at both time points, showed significant associations $(\mathrm{p}<0.001)$ for $\mathrm{WC}(\mathrm{OR}=1.06$ at $\mathrm{T} 0$ and $\mathrm{OR}=1.04$ at $\mathrm{T} 1)$ and $\mathrm{HDL}(\mathrm{OR}=0.98$ at $\mathrm{T} 0$ and $\mathrm{OR}=$ 0.98 at $\mathrm{T} 1)$ and the MetS score $(\mathrm{OR}=1.07)$ score at $\mathrm{T} 1$.

Discussion: The association between MetS and inflammation is already present in children. Out of the components of the MetS, WC and HDL were the ones more associated with an inflammatory state at two times points. Also the MetS score, but only at the follow-up, was associated with the hs-CRP. Therefore, in order to prevent the inflammatory state in childhood, efforts to improve the metabolic profile, specially WC and HDL, need to be made.
\end{abstract}

\section{Conflict of Interest}

There is no conflict of interest 